\title{
Experimental Characterization of a Hybrid Graphene/Metal Plasmonic Antenna Array
}

\author{
Arka Karmakar \\ University at Buffalo \\ Buffalo, New York \\ arkakarm@buffalo.edu
}

\author{
Farah Vandrevala \\ University at Buffalo \\ Buffalo, New York \\ farahper@buffalo.edu
}

\author{
Arjun Singh \\ University at Buffalo \\ Buffalo, New York \\ asingh29@buffalo.edu
}

\author{
Josep M. Jornet \\ University at Buffalo \\ Buffalo, New York \\ jmjornet@buffalo.edu
}

\author{
Erik Einarsson \\ University at Buffalo \\ Buffalo, New York \\ erikeina@buffalo.edu
}

\begin{abstract}
We fabricated terahertz-frequency plasmonic antenna arrays using patterned metallic structures atop a layer of graphene. The antenna design was developed through numerical simulations that were informed by experimentally obtained graphene parameters. Experimental characterization reveals a clear reflection enhancement at $3 \lambda / 2$, the intensity of which was modified by underlying graphene.
\end{abstract}

\section{CCS CONCEPTS}

- Hardware $\rightarrow$ Plasmonics; Emerging tools and methodologies; Wireless devices;

\section{KEYWORDS}

Terahertz-band communications, graphene, two-dimensional materials, plasmonic antennas, antenna arrays

\section{ACM Reference Format:}

Arka Karmakar, Farah Vandrevala, Arjun Singh, Josep M. Jornet, and Erik Einarsson. 2018. Experimental Characterization of a Hybrid Graphene/Metal Plasmonic Antenna Array. In NANOCOM '18: NANOCOM '18: ACM The Fifth Annual International Conference on Nanoscale Computing and Communication, September 5-7, 2018, Reykjavik, Iceland. ACM, New York, NY, USA, 2 pages. https://doi.org/10.1145/3233188.3233222

\section{INTRODUCTION}

The more bandwidth becomes available, the more data we want to transfer and the more devices we wish to connect; and we want to do all of this wirelessly. This insatiable hunger has been driving technology, resulting in a doubling of wireless data rates approximately every 18 months. [3] This trend-known as Edholm's law of bandwidth-predicts Terabit-per-second (Tbps) data rates within a few years. [7] However, just as Moore's law has pushed the limits of silicon technology, Edholm's law is pushing the limits of the

Permission to make digital or hard copies of all or part of this work for personal or classroom use is granted without fee provided that copies are not made or distributed for profit or commercial advantage and that copies bear this notice and the full citation on the first page. Copyrights for components of this work owned by others than the author(s) must be honored. Abstracting with credit is permitted. To copy otherwise, or republish, to post on servers or to redistribute to lists, requires prior specific permission and/or a fee. Request permissions from permissions@acm.org.

NANOCOM '18, September 5-7, 2018, Reykjavik, Iceland

(c) 2018 Copyright held by the owner/author(s). Publication rights licensed to ACM. ACM ISBN 978-1-4503-5711-1/18/09..\$15.00

https://doi.org/10.1145/3233188.3233222 wireless spectrum. In order to realize Tbps links, we are developing new technologies by incorporating plasmonic materials to access the currently unutilized terahertz band.

The major challenge to operating in the $\mathrm{THz}$ band $(0.1 \mathrm{THz}$ to $10 \mathrm{THz}$ ) is significant path loss, which is primarily due to absorption by water vapor. Improving $\mathrm{THz}$ sources and detectors is one way to overcome this problem, and there have been considerable advances in recent years. [1, 8] In addition to increased detector sensitivity and emitter power, directional antenna arrays are necessary to concentrate and steer the transmitted signals. Consequently, there is a critical need for compact antenna arrays capable of transmitting and receiving information in the $\mathrm{THz}$ band. In order to achieve this goal, control of the array response is essential.

Here we report measurements of a hybrid graphene/metal antenna array consisting of metallic elements fabricated atop graphene, which is a two-dimensional carbon allotrope with many impressive properties [4] and supports THz-frequency plasmons at room temperature. [2] The Fermi energy of graphene can be easily tuned by electrostatic gating, so graphene/metal hybrid structures are an important step toward controllable $\mathrm{THz}$ reflectarrays.

\section{FABRICATION}

We grew graphene on copper foil by chemical vapor deposition (CVD) at $1000{ }^{\circ} \mathrm{C}$ using methane as a carbon source. Graphene growth on copper is self-terminating, [6] resulting in continuous, primarily monolayer coverage over an area limited, in principle, by the size of the furnace and area of the foil. We used a piece of copper foil approximately $15 \mathrm{~mm} \times 15 \mathrm{~mm}$, and transferred the resulting graphene using a combination of poly(methyl methacrylate) (PMMA) and a compatible copolymer [5] onto a silicon substrate with $300 \mathrm{~nm}$ oxide layer. After transfer, we patterned gold antennas atop the graphene using electron-beam lithography. Antenna dimensions and spacing were determined by COMSOL Multiphysics simulations. Graphene parameters used in the simulations were determined from the complex conductivity, which we extracted using time-domain terahertz spectroscopy. [9] Simulation results are shown in the upper left frame of Figure 1, and the upper right part of the same figure shows an optical image of the fabricated antenna array. Raman spectra taken before and after transfer show no $D$ peak near $1350 \mathrm{~cm}^{-1}$, indicating the graphene is of high quality. Intensity of the $2 \mathrm{D}$ peak (near $2700 \mathrm{~cm}^{-1}$ ) roughly double that of the $\mathrm{G}$ peak (near $1590 \mathrm{~cm}^{-1}$ ) indicates monolayer graphene. 

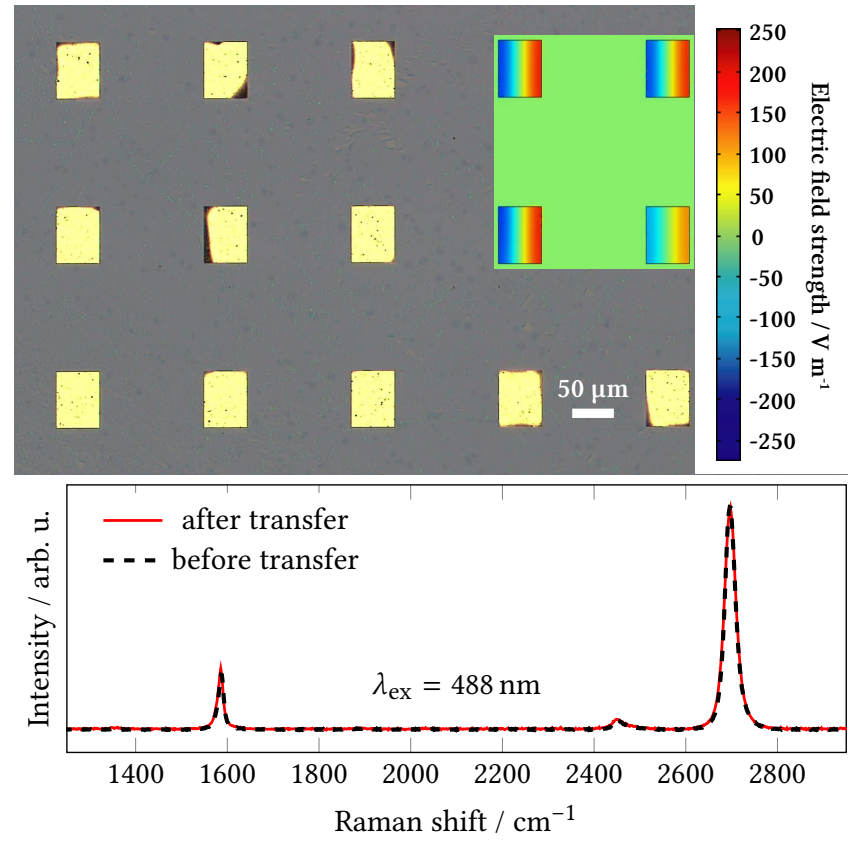

Figure 1: (Top) Optical micrograph showing an array of gold antennas fabricated atop graphene. Inset shows a numerical simulation of electric field response in a $2 \times 2$ antenna array. Scale bar applies to inset as well. (Bottom) Normalized Raman spectra obtained before and after graphene transfer.

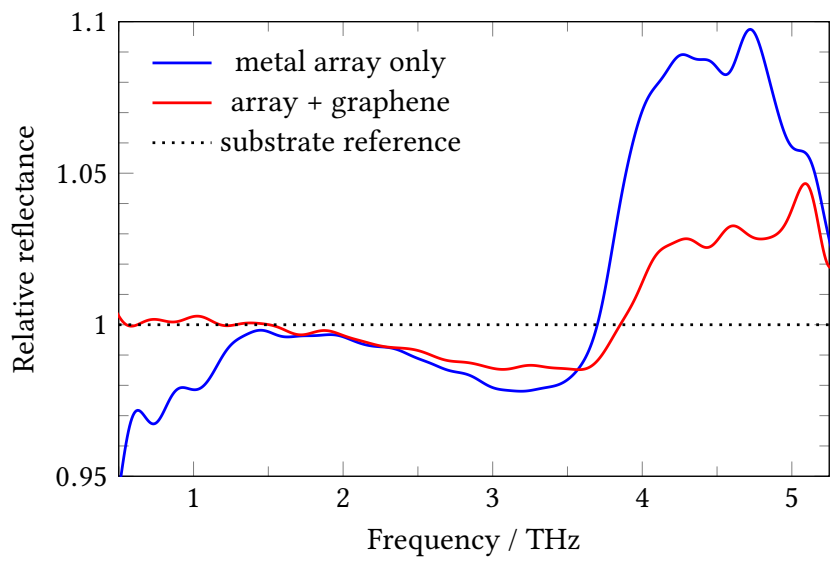

Figure 2: THz reflectance spectra relative to the underlying substrate. Increased reflectance by the array is apparent at 4.5 THz and is weakened by the presence of graphene.

\section{RESULTS AND DISCUSSION}

In Figure 2 we plot the response of the graphene/metal hybrid antenna array shown in Figure 1, as well as a metal array with identical dimensions but no underlying graphene. Reflectance is calculated relative to the underlying substrate reference, thus a value larger than unity corresponds to enhanced reflection by the array. The array elements were designed to resonate near $1.5 \mathrm{THz}$.
While we observe no change at $1.5 \mathrm{THz}$, the reflectance is clearly enhanced at $4.5 \mathrm{THz}$, corresponding to resonance at $3 \lambda / 2$. The lack of a response at $\lambda / 2$ is under investigation, but imperfect periodicity caused by errors in the fabrication process may have caused the primary reflection to be oriented away from our detector.

The decrease in reflectance when graphene is present can be understood by considering that, while it is a good conductor, graphene typically has a small density of states at the Fermi level. Having graphene on the surface under the array softens the electronic boundary condition at each element, making them slightly "leaky." The result is suppressed reflection at the boundary and reduced overall reflected power. This is advantageous because it facilitates modulation of the array response because the Fermi energy of graphene-and by extension the charge carrier concentration and electrical conductivity-can be tuned by electrostatic gating. The effect of such modulation would soften or sharpen the boundary, thereby changing the plasmon reflection from the edges and, consequently, the reflected power. Modulation of this hybrid array is currently being studied.

\section{CONCLUSION}

We designed and fabricated a hybrid reflect array comprised of metal elements atop graphene. Experimental measurements show a clear reflectance at $4.5 \mathrm{THz}$ that is modified by the presence of graphene. This result opens the door to control of the reflectarray response by electrostatic gating of the graphene. Moreover, the use of CVD for graphene synthesis combined with array fabrication by standard lithography suggests excellent potential to scale the array dimensions and number of elements for the purpose of manufacturing large scale reflectarrays.

\section{ACKNOWLEDGMENTS}

This work was supported by the Air Force Office of Scientific Research (AFOSR) under Grant FA9550-16-1-0188.

\section{REFERENCES}

[1] Robert Bogue. 2018. Sensing with terahertz radiation: a review of recent progress. Sensor Review 38, 2 (2018), 216-222. https://doi.org/10.1108/SR-10-2017-0221

[2] A. H. Castro Neto, F. Guinea, N. M. R. Peres, K. S. Novoselov, and A. K. Geim. 2009. The electronic properties of graphene. Rev. Mod. Phys. 81 (Jan 2009), 109-162. Issue 1. https://doi.org/10.1103/RevModPhys.81.109

[3] S. Cherry. 2004. Edholm's law of bandwidth. IEEE Spectrum 41, 7 (July 2004), 58-60. https://doi.org/10.1109/MSPEC.2004.1309810

[4] A. K. Geim and K. S. Novoselov. 2007. The rise of graphene. Nature Materials 6, 3 (March 2007), 183-191.

[5] Arka Karmakar, Farah Vandrevala, Florian Gollier, Mahima Ann Philip, Simran Shahi, and Erik Einarsson. 2018. Approaching completely continuous centimeterscale graphene by copolymer-assisted transfer. RSC Adv. 8 (2018), 1725-1729. Issue 4. https://doi.org/10.1039/C7RA12328K

[6] Xuesong Li, Weiwei Cai, Jinho An, Seyoung Kim, Junghyo Nah, Dongxing Yang, Richard Piner, Aruna Velamakanni, Inhwa Jung, Emanuel Tutuc, Sanjay K. Banerjee, Luigi Colombo, and Rodney S. Ruoff. 2009. Large-Area Synthesis of HighQuality and Uniform Graphene Films on Copper Foils. Science 324, 5932 (2009), 1312-1314. https://doi.org/10.1126/science.1171245

[7] A. Moldovan, S. Kisseleff, I. F. Akyildiz, and W. H. Gerstacker. 2016. Data rate maximization for terahertz communication systems using finite alphabets. In 2016 IEEE International Conference on Communications (ICC). 1-7. https://doi.org/10. 1109/ICC.2016.7511275

[8] Wendao Xu, Lijuan Xie, and Yibin Ying. 2017. Mechanisms and applications of terahertz metamaterial sensing: a review. Nanoscale 9 (2017), 13864-13878. Issue 37. https://doi.org/10.1039/C7NR03824K

[9] Yixuan Zhou, Yiwen E, Lipeng Zhu, Mei Qi, Xinlong Xu, Jintao Bai, Zhaoyu Ren, and Li Wang. 2016. Terahertz wave reflection impedance matching properties of graphene layers at oblique incidence. Carbon 96 (2016), 1129-1137. 